\title{
Combining IoT Deployment and Data Visualization: experiences within campus maintenance use-case
}

\author{
Rumana Yasmin \\ centre for wireless \\ communications \\ university of oulu \\ Oulu, Finland \\ rumana.yasmin@oulu.fi
Konstantin Mikhaylov
centre for wireless
communications
university of oulu
Oulu, Finland u.fi \\ konstantin.mikhaylov@oul \\ Susanna Pirttikangas \\ center for ubiquitous \\ computing \\ university of oulu \\ Oulu, Finland \\ susanna.pirttikangas@oulu \\ .fi
}

\author{
Miikka Salminen \\ center for ubiquitous \\ computing \\ university of oulu \\ Oulu, Finland \\ miikka.salminen@oulu.fi \\ Minna Pakanen \\ center for ubiquitous \\ computing and interact \\ research unit \\ university of oulu \\ Oulu, Finland \\ minna.pakanen@oulu.fi \\ Ari Pouttu \\ centre for wireless \\ communications \\ university of oulu \\ Oulu, Finland \\ ari.pouttu@oulu.fi
}

\author{
Ekaterina Gilman \\ center for ubiquitous \\ computing \\ university of oulu \\ Oulu, Finland \\ ekaterina.gilman@oulu.fi \\ Arttu Niemelä \\ center for ubiquitous \\ computing \\ university of oulu \\ Oulu, Finland \\ arttu.niemelä@oulu.fi
}

\author{
Juha Petäjäjärvi \\ centre for wireless \\ communications \\ university of oulu \\ Oulu, Finland \\ juha.petäjäjärvi@oulu.fi \\ Jukka Riekki \\ center for ubiquitous \\ computing \\ university of oulu \\ Oulu, Finland \\ jukka.riekki@oulu.fi
}

\begin{abstract}
The internet of things (IoT) application domain is evolving continuously and is expected to bring digitalization making everything smart. This paper presents reference architecture for IoT system. Moreover, full implementation of such architecture is demonstrated with the IoT testbed deployed at the University of Oulu. Leveraging MQTT protocol, LoRaWAN is utilized as an IoT enabler technology for our platform, comprising a cloud server as well. In addition, a hybrid server is added to the platform to enable enhanced performance and functionality. To showcase the potential impacts of augmented reality (AR) based applications in an IoT infrastructure, we implemented and tested the maintenance support application. The implemented application visualizes environmental conditions and the sensor node itself as an augmented object on a mobile phone. A user study is carried out to discuss and showcase the potential impacts of using such a visualization approach.
\end{abstract}

Keywords - internet of things; augmented reality; information visualization; LPWAN; LoRaWAN; mMTC

\section{INTRODUCTION}

Fostering innovation and digitalization, internet of things (IoT) applications have implications in almost every aspect of human life. Prominent examples include smart cities, transportation, and healthcare [1]. To achieve its goals, IoT system needs diverse kinds of devices, sensors, and actuators deployed, for example in homes, hospitals, and universities. Collecting, analyzing, and presenting the insights from the data collected from these diverse devices is challenging. Although, prior IoT research and industry communities have paid great attention to IoT devices deployment, real-time visualization on mobile phone received limited research attention.

Dashboards are the most commonly used way of visualizing IoT data today, however, visualizing this information with an augmented reality (AR) [2] application may bring number of benefits to the management and optimization of services. The AR visualization enables showing the IoT information on top of the real world. The benefits of utilizing AR for information visualization in IoT applications is that in addition to delivering real-time information, it also creates connection between virtual and physical worlds. Using AR technology for information visualization of IoT data can help in improving, for example, the maintenance of the large network of sensors/devices.

The number of IoT architectures have been proposed in earlier research projects [1]. The steadily increasing number of new use cases require significant improvements in existing IoT architecture to cope with the expected digitalization trend. Nowadays, two primary categories of IoT-grade communication technologies such as massive machine type communication (mMTC) and ultra-reliable low latency communication are coming forward. As we show in this paper, development and deployment of IoT platform, is a nontrivial task, which can be implemented in various ways. In addition, AR applications for campus navigation and visualization of machine operation in industries, have already been implemented and tested [4]-[6]. These papers studied different aspects of transmitting sensor data with diverse underlying IoT technologies. LoRa wide area network (LoRaWAN), one of promising technology in this digitized trend, follows Aloha protocol designed for long-range communications; that simplifies media access control layer. However, random access may bring collision when large number of LoRa sensor nodes transmit data simultaneously. Therefore, application of LoRaWAN is exacerbated for mMTC rather than critical communications [7]. Our developed IoT platform is equipped with large-scale LoRaWAN and mobile AR application data visualization capabilities. We find this combination beneficial for upcoming IoT trend, whilst providing the new type of campus maintenance services for the user. To achieve that, we added local server to the platform. This server improves performance and enriches service functionality, as it, following the edgecomputing paradigm, brings use case closer to the end user. 
The architecture is supposed to non-critical mMTC which in principle is not delay sensitive [7]. Therefore, this addition will not be a bottleneck for use cases able to handle few milliseconds of delay.

Prior research has not focused much on end users. It has been investigated how people are made aware of IoT infrastructure available in the environment and how to deliver the IoT information in understandable and convenient manner [1]. Moreover, despite the rich attention from community to IoT research, sharing of results of concrete use cases implementing the real-world IoT system is rare as well. Therefore, this paper reports on the design and development of IoT system for Tellus Innovation Arena spaces in University of Oulu designed to make connections and enable cooperative work. First, we describe the reference architecture with adaptation of hybrid schema for such use case. Then, we provide details on system design and implementation. Finally, we introduce and analyze our AR application built on top of the designed IoT system.

The rest of the paper is structured as follows. Section II reviews the related work, section III presents reference architecture and section IV discusses its implementation. Afterwards, section $\mathrm{V}$ presents $\mathrm{AR}$ application and its evaluation. Section VI discusses the results of the conducted user study. Finally, section VII presents conclusion of the paper.

\section{RELATED WORK}

Several surveys identify different frameworks for IoTbased applications [8],[9]. Mostly, IoT frameworks are delivered as middleware solutions including device management, platform portability, context-awareness, security, and privacy properties. Such surveys explore IoT revolution, which comprises of emerging IoT sensor networks, communication technologies, internet and application protocols. Finally, Al-Fuqaha et al. [8] provide technical details of different IoT architectures and corresponding prototypes that deliver desired services. Daneels et al. [9] propose IoT platform which can serve both Low-power Wireless Personal Area Network and multi-hop Wireless Sensor Network technology. Initial hardware and software prototype for this IoT architecture was presented. Their article provides the performance evaluation results by comparing these two technologies in different situations such as power consumption, RSSI, throughput, and latency.

Typically, the IoT indoor monitoring systems deliver information to their users via dashboards through Web portals or mobile applications [3],[10]. Such solution provides greater opportunities for analysis of both real-time and historical information that can be accessed from anywhere. In addition, notification mechanisms could be integrated into the dashboard [10]. Ahrabian et al. [11] provide a case study, where analysis of IoT sensor data, consisting of a CityPulse data set, is enabled through a web application, requiring no installation of additional tools by the end-user. The analysis, which the user can request by combining different algorithms in an HTTP request, is performed on the web server [11].

AR technology allows supplementing the real world view with additional generated content, like images, and sensor data [6]. Such content supplement is based on visual markers, GPS location or analysis of the images of the place. White and Feiner [12] present SiteLens system visualizing relevant

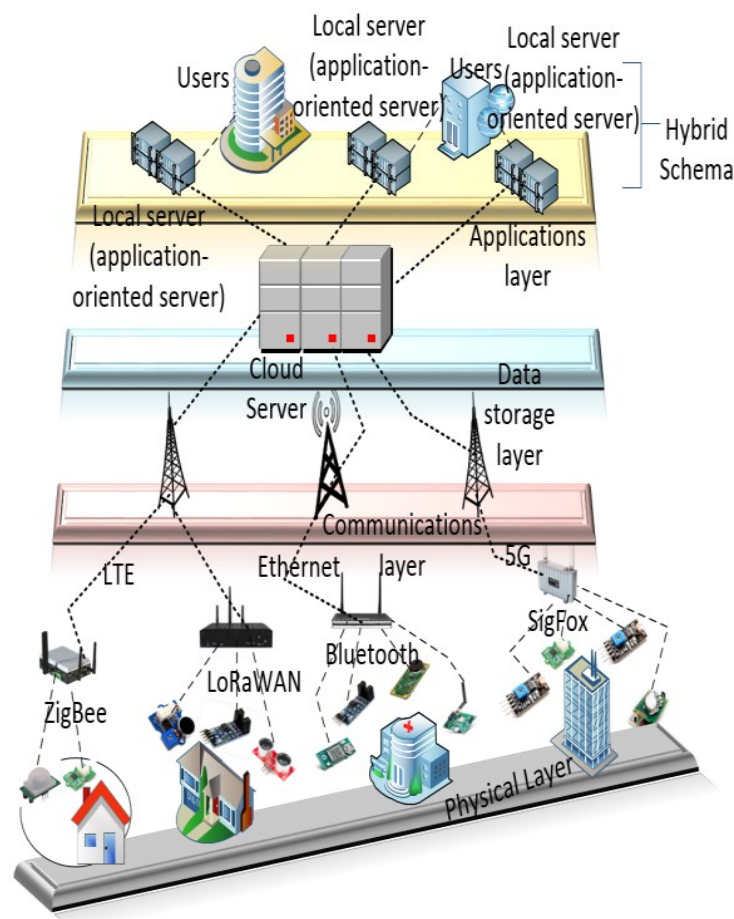

Fig. 1. Reference IoT system architecture.

virtual data directly in the context of the physical site. Other examples include SmartSantander project that has developed the AR application allowing real time access to traffic and beaches cameras, weather reports and forecast, public buses information and bike-rental service [13]. Also, [14] use AR for environmental monitoring service ekoNET targeting wide citizen population using the serious gaming concept.

\section{APPLICATION-ORIENTED IOT ARCHITECTURE}

Based on analysis of related research, we present traditional layered approach for our IoT architecture shown in Fig. 1. Such layered approach provides number of advantages, such as separation of concerns and clear data flow model [15]. The lowest, physical layer provides mechanisms to equip the space with physical devices, i.e., things. Communication layer offers means to deliver information from things to Internet. Data storage layer is responsible for storing and it performs general data pre-processing tasks. Application layer, generally, analyses data for use cases. In addition, we suggest to include a local server to achieve the benefits of hybrid schema. In this paper, under the term hybrid schema we mean exploitation of application-oriented servers along with the cloud service. Similarly to the edge computing, we aim to bring 'cloud' functionality to the edge of the network, closer to the end user, for more efficient data access [16]. Note that addition of an extra server as part of IoT architecture in some cases may bring delays, e.g., when the message should go from the end-user to the core components. The details of the implemented IoT architecture, from physical to application layer, are described in the following subsections.

\section{A. Physical layer}

Physical layer is formed by sensor nodes and the IoT gateways. The potential solutions for IoT on the physical layer ranges from, e.g., LPWAN, ZigBee, Wi-Fi, Bluetooth to LTE$A$ and 5G-MTC [8],[9]. However, LPWAN is being the dominant form of long-range wireless network for IoT 
devices. [7]. In contrast, some of these have been limited by the distance; some of these are currently limited due to technology immaturity. Although IoT-enabled technologies offer similar services, they vary in terms of technical capabilities, specifications, data rate, communication range capacity, quality of service support. Therefore, one of the primary challenges is to analyze wireless sensor network options before deployment for intended application.

\section{B. Communications layer}

The communication layer of the IoT platform is responsible for connecting sensor networks with Internet. Considering this layer within IoT paradigm, the sensor technologies such as Wi-Fi, NB-IoT or 6LoWPAN enable communication support IP from device-level. The LoRaWAN or Bluetooth use non-IP communication between device and gateway and thus require gateway to convert to IP. To enable IP support, the state-of-the-art cellular networks such as LTE and the incoming 5G look especially attractive as a backbone connectivity solution [17]. Alternatively, as the means for backbone connectivity can be used such technologies as $\mathrm{Wi}$ Fi or even wired connectivity solutions (e.g., Ethernet).

\section{Data storage layer}

The data storage layer consists of cloud storage backend to store and process data from physical devices. The cloud infrastructure, as part of the data storage layer in an IoT architecture, primarily comprises of platform-as-a-service clouds to store and process data. A Cloud platform is a specialized event-service database designed to provide seamless connectivity with any network environment irrespective of its location. Cloud services typically support either one or more communication protocols and predefined software tools e.g., message queueing telemetry transport (MQTT), constrained application protocol [17].

\section{Applications layer}

This layer performs data management and analysis tasks within concrete applications. An extra server in the application layer is formatted with a cloud data storage. This server is located closer to the user and provides similar functionality as that of cloud data centre, so called hybrid schema in this paper. Implementing hybrid schema by adding an applicationoriented server could make the data from a cloud accessible to a user just at the edge of ones' own network application layer. Moreover, using this mechanism, server could also be implemented locally ensuring hardware and software compatibility for specific devices and scalability for different use cases. The advantages of such design are:

- Provisioning of data management and storage layer for IoT data which can be optimized according to the needs of the use case.

- Deployment of services very close to the end user, reducing latency and improving the performance.

- Contrary to commercial clouds, this layer, as a storage entity and an application development platform, could be utilized as an open playground for innovative IoT developments.

- The application programming interfaces (APIs) to the applications in this layer could be shared to research partners and remote access could be granted, consortium partners could work together on a single platform to yield remarkable results.

\section{IMPLEMENTED INFRASTRUCTURE}

In our implementation, physical layer consists of 331 sensing devices placed into the Tellus Innovation Arena at University of Oulu premises. Each sensor device measures temperature, $\mathrm{CO} 2$, humidity, light, and motion and reports these data every 15 minutes. The reason behind selection of this report time - slow change in value of the mentioned parameters and - it reduces packet collisions. The data are transferred via LoRaWAN technology and collected by a gateway. This technology is currently the most widely available one. The network utilizes unlicensed sub-GHz ISM band [17] due to improved propagation characteristics, in particular for indoor environment. Based on the applications, it can manage large coverage support by adjusting spreading factor (SF), bandwidth and bit rate. In our scenario, we utilize SF 7 to reach optimal power consumption with $125 \mathrm{kHz}$ bandwidth. For further network deployment details and practical challenges refer to [18].

To get the data from the sensors, a single gateway Multitech Conduit - is used. The gateway supports LoRaWAN class ' $A$ ' functionality. The network server component of LoRaWAN is located right on the gateway. Additionally, the gateway supports MQTT protocol on top of the TCP protocol, which allows communication between network server and the cloud platform. This constitutes the communication layer for our deployment.

All the accumulated sensor data are stored on the ThingWorx cloud platform [19], which represents the data storage layer. ThingWorx data can be queried through proprietary HTTP-based APIs. The details about the performance of the physical and communication layers are available in [18].

Application layer performs both data analysis and management tasks. It consists of several components. First component utilizes an automated Python script that periodically pulls new data from the ThingWorx platform and stores it in a PostgreSQL database. Additional information, relevant for the analysis is also stored, including geopositioned location information of each sensor. Also, components performing actual analysis are implemented with scripts. Finally, a RESTful service is deployed to provide access to the data and corresponding analysis results and visualizations. Such approach provides a convenient and secure data access to the client applications, e.g. application might be interested to fetch data from a particular location or time period. We use JSON for HTTP payload when importing data from the ThingWorx platform. The REST API has been implemented with Django REST Framework and data visualization with Matplotlib.

Next, an illustrative application, which relies on the aforementioned RESTful service, was implemented. Our platform provides opportunity to implement AR-based visualization of sensor information. Such functionality is implemented using Unity framework with an AR plugin Alvar, providing marker-, multi-marker, 2D image- and 3D point cloud-based tracking [19]. Therefore, we provide augmented visualization for custom location. 


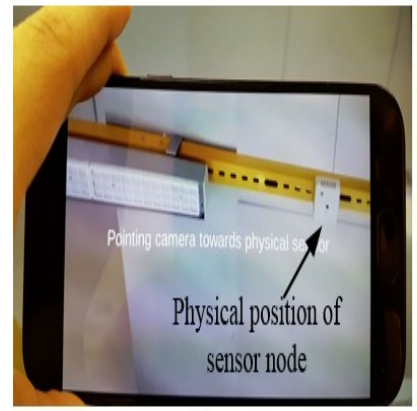

(a)

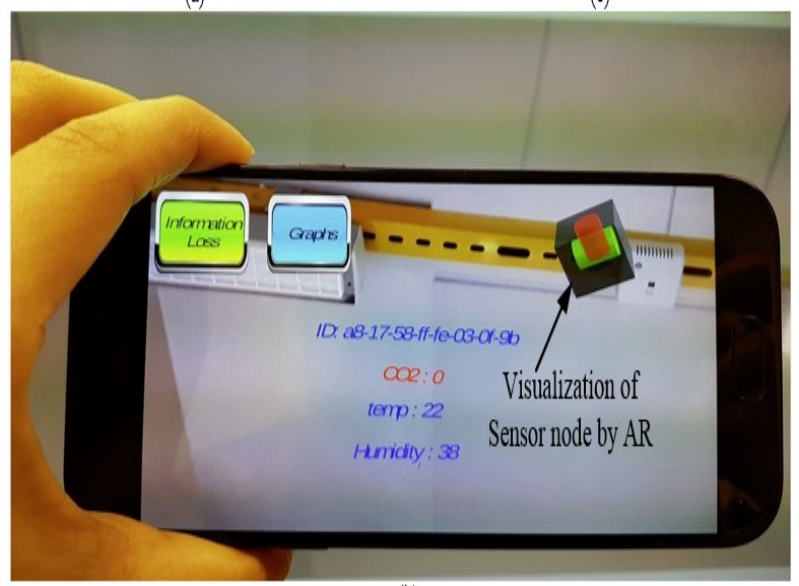

(b)

Fig. 3. TellusAR application on mobile device. User starts the application and points the phone's camera to the sensor node (a). Application recognizes the sensor and demonstrates its data over the camera view (b). Finally, user can see the weekly statistics for the recognized sensor (c).

\section{Augmented Reality Use Case: Maintenance}

\section{A. Use Case}

Real world sensor installations are prone to errors, faults, and biases. Therefore, to test our platform and gather some information on its utility, we decided to implement application that helps in locating the faulty sensors for maintenance. We developed the AR mobile application on top of the proposed IoT infrastructure. To provide more natural user experience, in addition to the dashboard, we utilize AR to show the sensor data. The application shows sensor specific information such as time-series graphs of the measured environmental parameters or their current values (refer to Fig. 2 for an illustration). The erroneous values would be blinking when the phone is pointed to the faulty sensor node. At the current stage, no navigation towards a faulty sensor is supported by the AR application.

\section{B. Methodology}

Experiment took place in Tellus Innovation Arena space of University of Oulu. User study participants were recruited randomly from the ordinary visitors of this public space. Overall, 14 persons participated in the study. The participants were given short introduction about the purpose of the study and overview of the functionality of the application. Then, participants tried the application by themselves until they felt comfortable with its operation. After that, participants were given two tasks in which they had to locate the faulty sensor in Tellus. In the first task, participants were asked to locate the faulty sensor by relying on the information shown on the

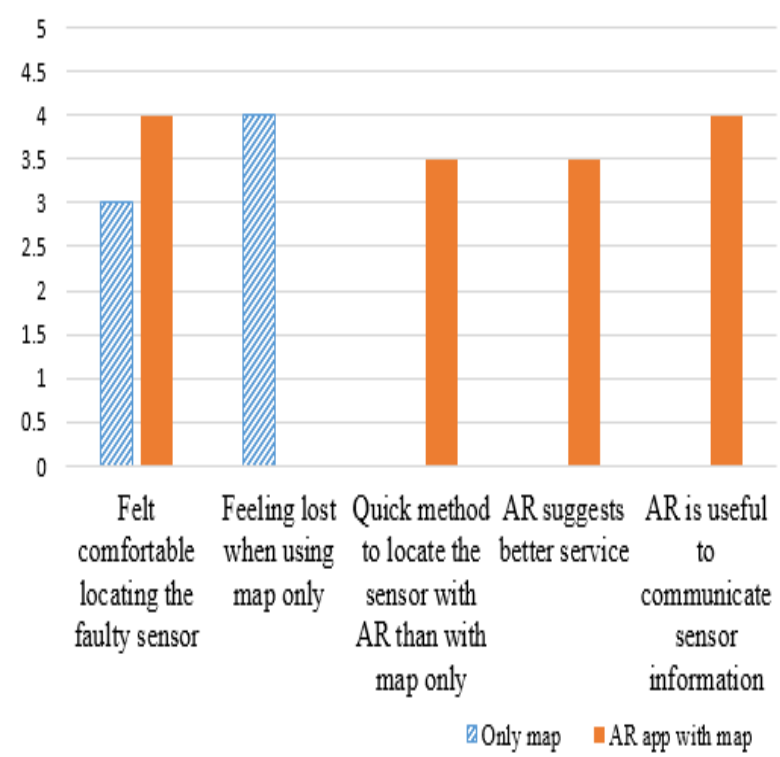

Fig. 2. The user study results showing the average scores of using only map and AR visualization application with map.

dashboard view which only showed the location of the sensor node on the Tellus map. In the second task, participants were asked to locate the faulty sensor by relying on both the location information of the faulty sensor and AR application, showing actual sensor values. Task completion time was measured, correctness of locating the faulty sensor, as well as behavior of the test users was observed. To reduce the bias, the order of the tasks was randomized for the participants. Participants were encouraged to comment aloud their feelings and concerns about the application. After the study, participants were asked to answer a questionnaire to get their feedback. The questionnaire had five questions: comfort of using the system, feeling lost when using the map only, time aspect when AR application is used, as well as overall service and usefulness of AR application. Each category was evaluated with the Likert scale from 0 to 5 , where 0 means fully disagree and 5 means strongly agree. Questionnaire also included open answer field, where participants could give general feedback about the system. In addition, we asked each participant to choose the preferred method for a task completion.

\section{Results}

Results from the questionnaire are presented in Fig. 3. Here, we can see that feeling of comfort is better for the AR application, when compared to the map only condition. As well as feeling of lost for the map only condition is rather agreed by the users. This is also supported by the fact that with map only version, only $50 \%$ of participants correctly located the faulty sensor, whereas $90 \%$ of test users were able to find the faulty sensor with AR application. Mostly, participants agreed that with AR application it is quicker to locate the sensor than compared to the map only, however, for AR application itself it took some time to distinguish between sensors, as they all look the same. Therefore, AR approach intends to suggest better service in the future. In addition to usefulness, the participant found the presentation of sensor data by means of $A R$ rather convenient. 
Observation during a user study revealed that participants required more time to locate the faulty sensor with $A R$ application, however, this was due to response time of $A R$ application, coming from the challenge of distinguishing between the identical sensors. Our AR application relied on 3D point cloud -based tracking (no tags were placed on the sensor nodes) [19], therefore it took some time for the application to distinguish the correct one. Most users chose AR application as their preferred method for task completion. This was expected outcome, as the deployed sensors are identical and are placed in a grid with two meters apart from each other, which makes it hard to distinguish the faulty one using just the map.

\section{CONCLUSIONS}

The paper reports on the design and evaluation of combination of LoRaWAN network with AR visualization. To study the utility of such combination in a close-to-life scenario, we instrument a practical application addressing the sensor infrastructure maintenance use case. The designed application helps to locate faulty sensors and keep track of data accuracy. The architecture was deployed in University of Oulu and exemplified with AR application.

The real time data from sensors were visualized with mobile phone application with the augmented camera view, improving the user experience. This application was fully implemented and tested with voluntary users. The conducted user study provided user feedback on application in terms of comfort of using the system, feeling lost when using the map only, time aspect when AR application is used, as well as overall service and usefulness of AR application. Analysis of the user study reveals the utility of such an application in service maintenance as an example. The developed application implements basic functionality with improvements expected to be added in the near future.

Future work includes obtaining and visualizing the more advanced information from sensors and providing capabilities for maintenance person to directly interact with the sensor through mobile application. We are also interested in other kinds of applications and possible ways of delivering information to the users.

\section{ACKNOWLEDGMENT}

This work has been partially funded by Business Finland and several companies through VIRPA $\mathrm{C}$ and $\mathrm{D}$. The acquisition of the hardware for this study has also been partially funded by the ERDF project A71720, 'Big Data for $5 \mathrm{G}^{\prime}$, governed by the HILLA program (www.hilla.center). This research work has been financially supported by Academy of Finland 6Genesis Flagship (318927) and by EU Horizon 2020 project CUTLER: Coastal Urban developmenT through the LEnses of Resiliency, under contract no. 770469 (http://www.cutler-h2020.eu/).

\section{REFERENCES}

[1] A. Zanella, N. Bui, A. Castellani, L. Vangelista and M. Zorzi, "Internet of Things for Smart Cities," IEEE Internet Things J. , vol. 1, pp. 22-32, Feb. 2014.

[2] L. Zhang, S. Chen, H. Dong and A. El Saddik, "Visualizing Toronto City Data with HoloLens: Using Augmented Reality for a City Model," IEEE Consum. Electron. Mag., vol. 7, pp. 73-80, May 2018.

[3] J. Shah and B. Mishra, "Customized IoT Enabled Wireless Sensing and Monitoring Platform for Smart Buildings,” in Proc. Int. Conf. Ind. Autom. Manuf. Eng., vol. 23, 2016, pp. 256-263.

[4] C. H. Lin et al., "A novel campus navigation APP with augmented reality and deep learning," IEEE Int. Conf. on Appl. Syst. Invention, 2018, pp. 1075-1077.

[5] J. Um, J. Popper and M. Ruskowski, "Modular augmented reality platform for smart operator in production environment," IEEE Ind. Cyber-Phy. Syst., 2018, pp. 720-725.

[6] H. Subakti and J. R. Jiang, "Indoor Augmented Reality Using Deep Learning for Industry 4.0 Smart Factories," IEEE 42nd Annu. Comput. Software and Appl. Conf., 2018, pp. 63-68.

[7] M. Tome, P. Nardelli and H. Alves, "Long-range Low-power Wireless Networks and Sampling Strategies in Electricity Metering," IEEE Trans. Ind. Electron., pp. 1-8, March 2018.

[8] A. Al-Fuqaha et al., "Internet of Things: A Survey on Enabling Technologies, Protocols, and Applications," IEEE Commun. Surveys Tut., vol. 17, pp. 2347-2376, 2015.

[9] G. Daneels et al., "Real-Time data dissemination and analytics platform for challenging IoT environments," in Proc. Global. Inf. Infrastructure Netw. Symp., 2017, pp. 23-30.

[10] G. Marques, and R. Pitarma, "An Indoor Monitoring System for Ambient Assisted Living Based on Internet of Things Architecture," Int. J. Environ Res Public Health, vol. 13, pp. 1152-1165, Nov. 2016.

[11] A. Ahrabian, S. Kolozali, S. Enshaeifar, C. Cheong-Took and P. Barnaghi, "Data analysis as a web service: A case study using IoT sensor data,"in Proc. Int. Conf. on Acoust., Speech and Signal Process., 2017, pp. 6000-6004.

[12] S. White and S. Feiner, "SiteLens: situated visualization techniques for urban site visits," in Proc. of the SIGCHI Conf. on Human Factors in Comput. Syst., 2009, pp. 1117-1120.

[13] Smart Santander FP7 project, "SmartSantanderRA - Santander augmented reality application," 2018, [Accessed: 2018-03-21]. [Online]. Available: http://www.smartsantander.eu/index. $\mathrm{php} / \mathrm{blog} /$ item/174-smartsantanderra-santander-augmented-realityapplication.

[14] B. Pokric, S. Krco and M. Pokric, "Augmented Reality Based Smart City Services Using Secure IoT Infrastructure," in Int. Conf. on Adv. Inform. Netw. and Appl., 2014, pp. 803-808.

[15] E. Gilman et al., "Perception framework for supporting development of context - aware web services," Int. J. of Pervasive Comput. and Commun., Vol. 7, pp.339-364, 2011.

[16] J. Ren, H. Guo, C. Xu and Y. Zhang, "Serving at the Edge: A Scalable IoT Architecture Based on Transparent Computing," IEEE Netw., vol. 31, pp. 96-105, 2017.

[17] R. Yasmin, J. Petäjäjärvi, K. Mikhaylov and A. Pouttu, "On the Integration of LoRaWAN with the 5G Test Network," in Proc. 28th Annu. Int. Symp. Personal, Indoor Mobile Radio Commun., 2017, pp. 1- 6.

[18] R. Yasmin, J. Petäjäjärvi, K. Mikhaylov and A. Pouttu, "Large and Dense LoRaWAN Deployment to Monitor Real Estate Conditions and Utilization Rate," in Proc. 29th Annu. Int. Symp. Personal, Indoor Mobile Radio Commun, 2018, in press.

[19] VTT, “Alvar", 2018, [Accessed: 2018-05-12]. [Online]. Available: http://alvar.erve.vtt.fi. 